\title{
Uretrostomia perineal em felino obstruído de três meses de idade: Relato de caso
}

\author{
Solimar Dutra da Silveira ${ }^{*}$, Liege Georgia Andrioli Martins ${ }^{2}$, Carla Sordi Furlanetto ${ }^{3}$, \\ Carolina Martins Lazarotto ${ }^{4}$, Eduarda Gabriela Mansour ${ }^{5}$, Alan Passador da Silva ${ }^{6}$ \\ ${ }^{1}$ Graduando do curso de Medicina Veterinária da Pontifícia Universidade Católica do Paraná - PUCPR, Toledo, PR - Brasil. E-mail: \\ solimards@hotmail.com \\ ${ }^{2}$ Docente do curso de Medicina Veterinária da Pontifícia Universidade Católica do Paraná - PUCPR, Toledo, PR - Brasil. E-mail: \\ liege.martins@pucpr.br \\ ${ }^{3}$ Médica Veterinária Residente em Clínica Médica e Cirúrgica de Pequenos Animais do Hospital Veterinário da Pontifícia Universidade Católica do \\ Paraná-PUCPR, Toledo,PR-Brasil.E-mail: carla.sordi@hotmail.com \\ ${ }^{4}$ Médica Veterinária Residente em Clínica Médica e Cirúrgica de Pequenos Animais do Hospital Veterinário da Pontifícia Universidade Católica do \\ Paraná - PUCPR, Toledo,PR - Brasil.E-mail: carolina_ml@hotmail.com \\ ${ }_{5}^{5}$ Médica Veterinária Anestesiologista do Hospital Veterinário da Pontifícia Universidade Católica do Paraná - PUCPR, Toledo, PR - Brasil. E-mail: \\ eduardamansour@gmail.com \\ ${ }^{6}$ Médico Veterinário Residente em Clínica Médica e Cirúrgica de Pequenos Animais do Hospital Veterinário da Pontifícia Universidade Católica do \\ Paraná - PUCPR, Toledo,PR - Brasil.E-mail: alan91_passador@hotmail.com \\ *Autor para correspondência
}

\begin{abstract}
RESUMO. O presente relato tem como objetivo descrever um caso de obstrução uretral em um felino macho, sem raça definida de três meses de idade. Suspeitou-se de um quadro obstrutivo durante a anamnese e exame físico, ao qual o paciente se encontrava apático, estado de desidratação leve e vesícula urinária distendida. Após realização de exames complementares, realizaram-se três tentativas de sondagem uretral com cateter intravenoso flexível, ambas demonstrando insucesso. O paciente então, foi encaminhado para cirurgia, procedendo-se amputação total peniana, orquiectomia e uretrostomia perineal; porém devido intercorrências anestésicas o paciente veio a óbito em virtude de duas paradas respiratórias e duas cardiorrespiratórias.
\end{abstract}

Palavras chave: Gato, obstrução, tampão uretral.

\section{Perineal urethrostomy in obstructed feline from three months old: Case report}

\begin{abstract}
The present report aims to describe a case of urethra lobstruction in a feline, male, without race defined three months of age. The suspected of a obstructive frame was during anamnesis and physical examination, to which the patient was lethargic, mildde hydration state and distended urinary bladder. After additional tests, were performed three triesurethral probing with intravenous catheter flexible, both failure demonstrating. The patient was referred for surgery, which amputation of the penis, orchiectomy and perineal urethrostomy. However, because of anesthetic complications the patient came to death in virtue of two stops respiratory and two cardio respiratory.
\end{abstract}

Keywords: Cat, obstruction, urethralplug

\section{Introdução}

Felinos machos anatomicamente caracterizamse por predisposição a obstruções de trato urinário inferior, sendo estas, significativamente ocasionadas por presença de urólitos e tampões uretrais, associado à diminuição do diâmetro luminal e da elasticidade da uretra peniana (Dibartola \& Westropp, 2015). De acordo com Belone (2002) uma obstrução urinária é definida

pela interrupção do fluxo da urina, independentemente do local de ocorrência, trazendo ao paciente, como consequência, uma suspensão na filtração renal.

Na clínica de pequenos animais, a obstrução uretral em felinos caracteriza-se como urgência ou emergência, sendo caracterizada como sinal 
clínico comum da doença do trato urinário inferior da espécie. A obstrução uretral associada a outras alterações geralmente levam o animal a um quadro de disúria ou anúria e em casos mais graves o animal pode vir a óbito (Rabelo et al., 2005; Serrano, 2011). Durante obstruções uretrais, severas disfunções metabólicas podem ser desencadeadas pela elevação de metabólitos proteicos como a ureia e creatinina, ocasionando quadros de azotemia pós-renal e pondo em risco a vida paciente. Alguns casos, lesões permanentes podem ocorrer devido acometimento do sistema cardiovascular, respiratório, renal e nervoso (Belone, 2002; Dibartola \& Westropp, 2015).

A severidade relacionada a obstrução uretral é observada em felinos afetados por mais de 36 horas onde apresentam quadros de intensa apatia, súria, anúria, hematúria, anorexia, êmese, desidratação grave, hálito urêmico e dor em região abdominal durante a palpação (Rabelo et al., $\underline{2005}$ ).

Etiologicamente, a ocorrência de obstrução do trato urinário inferior em machos, mais comumente está relacionada a formação de urólitos ou tampões uretrais (Souza, 1998; Dibartola \& Westropp, 2015). Fatores como alimentação, obesidade, castração, pouca atividade física, enfermidades e estresse podem influenciar na formação dos tampões e urólitos (Osborneet al., 1995; Rabelo et al., 2005). Outras causas podem ser atribuídas a obstrução, porém de menor ocorrência como neoplasias uretrais, vesicais e prostáticas, estenose uretral induzida por sondagem inadequada ou por uretroplastia funcional com falha na técnica cirúrgica, laceração ou esmagamento peniano, anormalidades anatômicas e disfunções neurológicas (Dibartola \& Westropp, 2015). O diagnóstico de obstrução uretral pode ser obtido entre a associação do histórico clínico, exame físico e a utilização de métodos complementares que auxiliam na abordagem subsequente do paciente. Exames de imagem como radiografia e ultrassonografia, métodos laboratoriais como urinálise, hemograma, bioquímica sérica e até mesmo eletrocardiograma constituem importante ferramenta para designar a evolução da afecção, e o prognóstico do paciente (Reche et al., 1998; Rabelo et al., 2005). O tratamento adotado para a obstrução uretral deve ser baseado no grau e no tempo de duração dessa obstrução. A obstrução total, quando não aliviada, pode levar o animal à morte em 72 horas (Peixoto, 1997; Pauluka, 2008). Durante o quadro obstrutivo, as principais complicações relacionadas são: desidratação, podendo evoluir a hipovolemia e choque; azotemia a qual desencadeia um quadro de acidose metabólica, hiperfosfatemia, hipercaliemia e hipocalcemia. Devido a tais complicações, preconiza-se como terapêutica uma primeira abordagem clínica, realizando a estabilização do paciente por correção da hipovolemia e hipercaliemia, utilizando método de cateterismo venoso com administração de fluidoterapia, controle de dor e sedação para posterior realização de procedimento de descompressão da vesícula urinária por meio de cistocentese e cateterização uretral para desobstrução (Lazzarotto, 2001; Alves, 2006).

Caso a abordagem terapêutica de desobstrução uretral por cateterismo não apresente êxito o tratamento cirúrgico é indicado (Bjorling et al., 2008). Em literatura, diversas intervenções cirúrgicas estão descritas (Corgozinho \& Souza 2004), sendo a técnica de uretrostomia perineal a mais recomendada, pela eficácia em redução de novos episódios de obstrução física no nível da porção distal da uretra, mas não trará nenhum benefício se a obstrução se localiza na porção média ou proximal (Gregory, 1987; Osborne et al., 1996; Peixoto, 1997; Bjorling et al., 2008). Recomenda-se a abordagem cirúrgica por técnica de uretrostomia perineal em felinos machos, portadores de lesões irreversíveis na mucosa uretral peniana e para gatos que apresentem obstrução uretral recorrente e não responsiva ao tratamento clínico (Bjorloinget al., 2008; Osborne et al., 1996; Gregory, 1987). Com o procedimento de reparação cirúrgica por uretrostomia perineal, a micção urinária retorna à normalidade dentro de 24 horas pós-operatório (Alves, 2006).

O presente relato tem como objetivo, descrever um caso clínico de obstrução uretral em um felino macho, com três meses de idade, sem raça definida, enfatizando os aspectos clínicos, métodos de diagnóstico e terapêutica empregada.

\section{Relato de caso}

Foi atendido no Hospital Veterinário da Pontifícia Universidade Católica do Paraná, campus Toledo - PR, um felino, macho, sem raça definida, com três meses de idade e massa corporal de $0,8 \mathrm{~kg}$.

Durante anamnese, o tutor relatou como queixa principal, apatia, anorexia, disúria com início a três dias e episódios de vômito desencadeados durante tentativas de micção. Ainda de acordo 
com o responsável, o paciente se apresentava em estado de normodipsia, normoquezia, ausência de ectoparasitas, não havia sido vacinado e vermifugado e sua alimentação baseava-se em ração seca a granel.

No exame físico geral do paciente, constatouse desidratação leve, mucosas normocoradas, tempo de preenchimento capilar de 2 segundos, temperatura de $37,5^{\circ} \mathrm{C}$ e frequência cardíaca de 200 batimentos/minuto. Em avaliação da cadeia linfática, verificou-se ausência de reativação de linfonodos submandibulares, pré-escapulares, axilares, inguinais e poplíteos. Por meio de palpação se constatou intenso desconforto abdominal e vesícula urinária repleta.

O paciente foi encaminhado para exame ultrassonográfico de região abdominal com o propósito de avaliar o trato urogenital suspeitando-se de um quadro obstrutivo. Os rins se apresentavam levemente aumentados sem demais alterações visíveis pela ecografia. Já a vesícula urinária se apresentava distendida e com presença de urólitos durante movimentação vesical realizada pelo avaliador.

Procedeu-se tentativa de coleta sanguínea para a realização de bioquímica sérica; porém, devido à pouca massa corporal do paciente e estado de desidratação leve, a quantidade amostral obtida para análise foi insuficiente. Em seguida, realizouse aplicação de neuroleptoanalgesia com cetamina (3 $\mathrm{mg} / \mathrm{kg}$ ) por via intramuscular, cateterismo venoso com estabelecimento de fluidoterapia e indução anestésica com propofol $(2 \mathrm{mg} / \mathrm{kg})$ por via intravenosa, para procedimento de cistocentese objetivando coleta de urina para exame de urinálise, descompressão vesical e posteriormente realização de sondagem uretral. $\mathrm{Na}$ urinálise, o aspecto físico demonstrou-se turvo; coloração avermelhada demonstrando hematúria; densidade de 1018 (referência 1015 a 1040); pH 8,0 (referência 5,5 a 7,5); ausência de glicose, corpos cetônicos, bilirrubina, urobilinogênio e nitrito. Na sedimentoscopia se verificou quantidade moderada de bactérias; ausência de células descamativas, cilindros e leucócitos até 3 por campo (referência de 0-3 por campo quando urina coletada por cistocentese).

Realizou-se três tentativas de sondagem uretral com cateter intravenoso flexível (calibre $1,1 \times 32$ $\mathrm{mm}$ - rosa; $0,9 \times 25 \mathrm{~mm}$ - azul e $0,7 \times 19 \mathrm{~mm}-$ amarelo) devido lúmen uretral do paciente ser extremamente reduzido impossibilitando a passagem de sonda convencional, onde ambas as tentativas não demonstraram sucesso quanto uma desobstrução, confirmando a presença de um tampão obstruindo totalmente o lúmen uretral. Baseado nas decorrências apresentadas pelo paciente, firmou-se o diagnóstico de obstrução uretral.

Uma vez que o paciente não estava se alimentando e apresentava-se desidratado, optouse por manter a fluidoterapia (Ringer lactato 300 $\mathrm{ml}$ ), suplementação intravenoso com glicose $50 \%$ e cloreto de potássio como indicado por literatura (Wakil et al., 2010). Empregou-se como tratamento ambulatorial a administração de dexametasona $(0,5 \mathrm{mg} / \mathrm{kg})$, enrofloxacina $(2,5$ $\mathrm{mg} / \mathrm{kg}$ ), cloridrato de tramadol $(2 \mathrm{mg} / \mathrm{kg})$ e dipirona $(25 \mathrm{mg} / \mathrm{kg})$ ambos por via subcutânea. O paciente foi preparado para cirurgia imediata (Figura 1), onde se procedeu à amputação total do pênis, orquiectomia, seguida por uretrostomia perineal (Fossum, 2015), porém ao final do procedimento, por intercorrências anestésicas, infelizmente o paciente veio a óbito ocasionalmente após duas paradas respiratórias e duas cardiorrespiratórias.

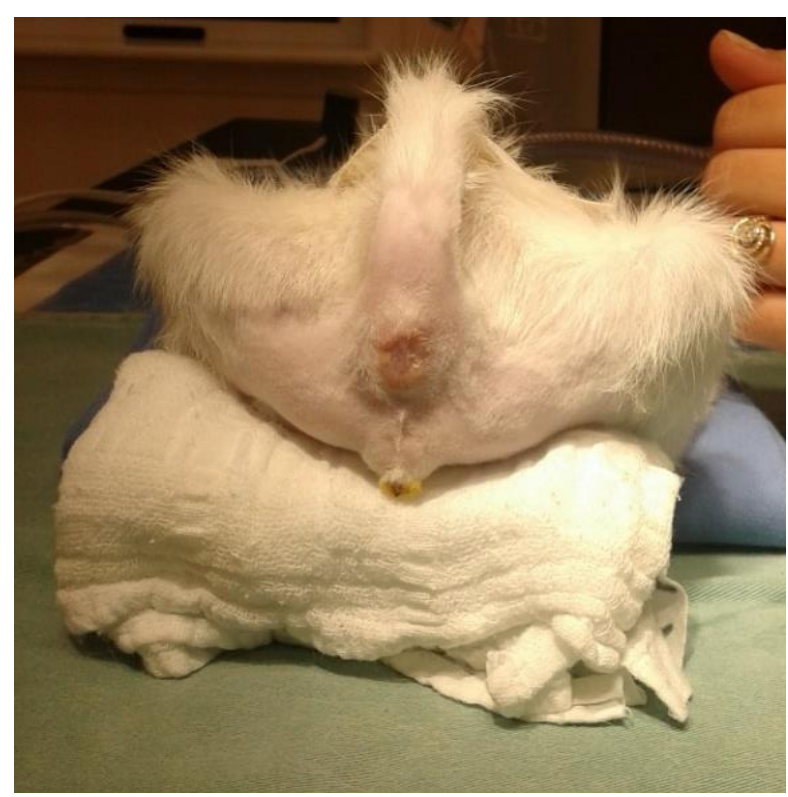

Figura 1. Felino macho, com três meses de idade, em decubito ventral, durante pré-cirurgico.

Inicialmente realizou-se a indução anestésica com propofol $(2 \mathrm{mg} / \mathrm{kg})$ via intravenosa, controle das vias aéreas com intubação endotraqueal e manutenção anestésica por inalação de isoflurano por circuito fechado e re-inalação. Posteriormente, procedeu-se antissepsia com gluconato de clorexidina sobre a região cirúrgica, demarcada previamente por tricotomia ampla em toda região 
abdominal, prepucial, escrotal e perineal (Figura 2). Durante este primeiro procedimento, se deu a primeira parada respiratória, onde foi realizado ressuscitação por estimulação do ponto de acupuntura "Jen Chung" com agulha hipodérmica, aumento do fornecimento de oxigênio $100 \%$ pelo aparelho de anestesia e administração intravenosa de adrenalina $(0,2 \mathrm{mg} / \mathrm{kg})$, obtendo sucesso e permitindo prosseguir com o procedimento cirúrgico, corroborando com protocolos descritos em literatura (Aquino Filho, 2014; Rossi et al., 2007).

A amputação do pênis (Figura 3) foi iniciada com dissecção do órgão e tecidos adjacentes, realizando anastomose dos vasos prepuciais, seguido por orquiectomia, sendo o tecido subcutâneo aproximado por sutura contínua simples utilizando fio absorvível 3-0. Posteriormente foi realizado a aproximação da pele por sutura descontínua e pontos simples com fio inabsorvível 4-0 (Fossum, 2015). Neste momento, o paciente apresentou uma segunda parada respiratória, a qual foi adotado os mesmos

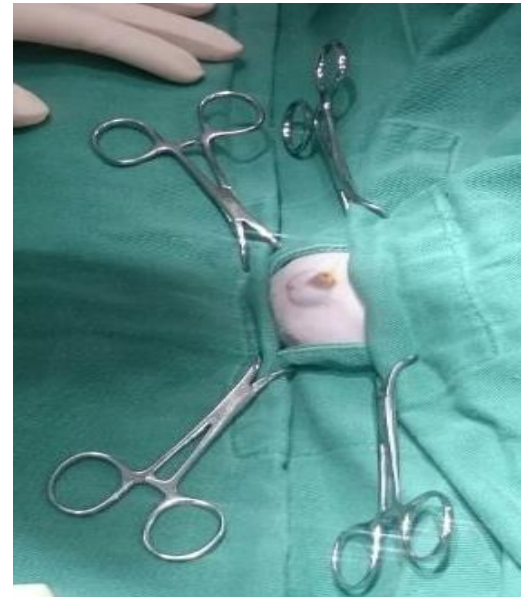

Figura 2. Felino macho, em decúbito dorsal durante inicio da intervenção cirúrgico.

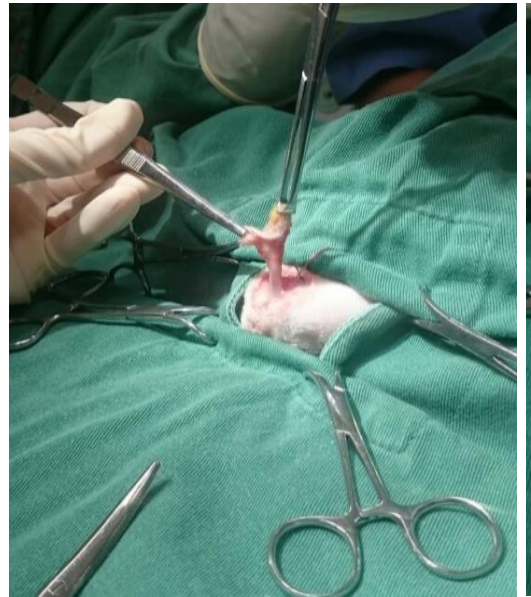

Figura 3. Paciente durante procedimento de dissecção do pênis e tecidos adjacentes para amputação peniana procedimentos de ressuscitação descritos anteriormente (Rossi et al., 2007).

Em seguida, realizou-se uretrostomia com auxílio de um cateter $(0,7 \times 19 \mathrm{~mm}$ - amarelo $)$ inserido parcialmente na uretra (Figura 4), o que permitiu a realização de uma incisão com o objetivo de aumentar a abertura do meato urinário que se estava criando (Fossum, 2015), onde ao realizar tal procedimento, encontrou-se a causa obstrutiva, sendo um tampão provavelmente formado a partir de urólitos (Figura 5 e 6). Ao final desta manobra cirúrgica, o paciente novamente apresentou desestabilização anestésica, ocasionando uma parada cardiorrespiratória. Adotou-se como procedimentos de ressuscitação massagem cardíaca, introdução de ventilação mecânica por ambu conectado a um cilindro de oxigênio $100 \%$, administração de atropina intravenosa $(0,04$ $\mathrm{mg} / \mathrm{kg})$ e adrenalina intracardíaca $(0,2 \mathrm{mg} / \mathrm{kg})$, estabilizando novamente o paciente e seguindo o procedimento cirúrgico com a ventilação mecânica (Rossi et al., 2007).

Fonte: Solimar Dutra da Silveira (2016).

Posteriormente, foi realizado sutura muco cutânea com pontos simples com fio inabsorvível 4-0. Ao final, o tecido subcutâneo e a pele cranial e caudal à uretrostomia foram suturados em duas camadas, como indicado em literatura (Fossum, 2015). Porém, durante a realização dos últimos pontos cirúrgicos, o felino novamente apresentou uma parada cardiorrespiratória, com nova tentativa de nova estabilização por ressuscitação, sem demonstrar sucesso, vindo o paciente à óbito.

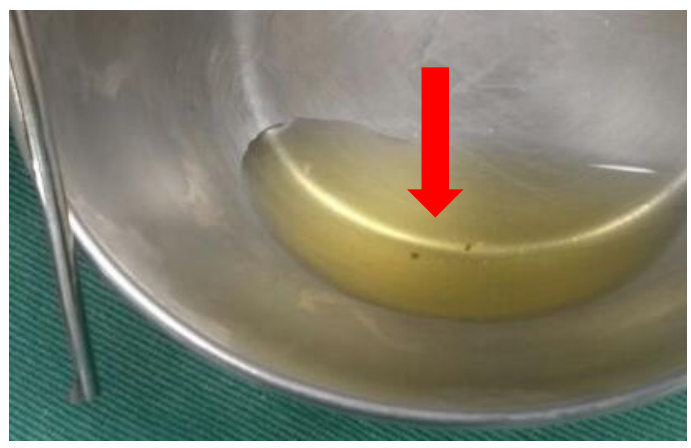

Figura 5. Urina coletada após sondagem uretral, demonstrando sedimentação (seta). 
A técnica cirúrgica adotada demonstrou sucesso e pode ser concluída (Figura 7), porém devido as intercorrências do procedimento anestésico, o paciente veio à óbito, sendo encaminhado ao setor de necropsia do Hospital Veterinário da Pontifícia Universidade Católica do Paraná, para posterior liberação ao tutor.

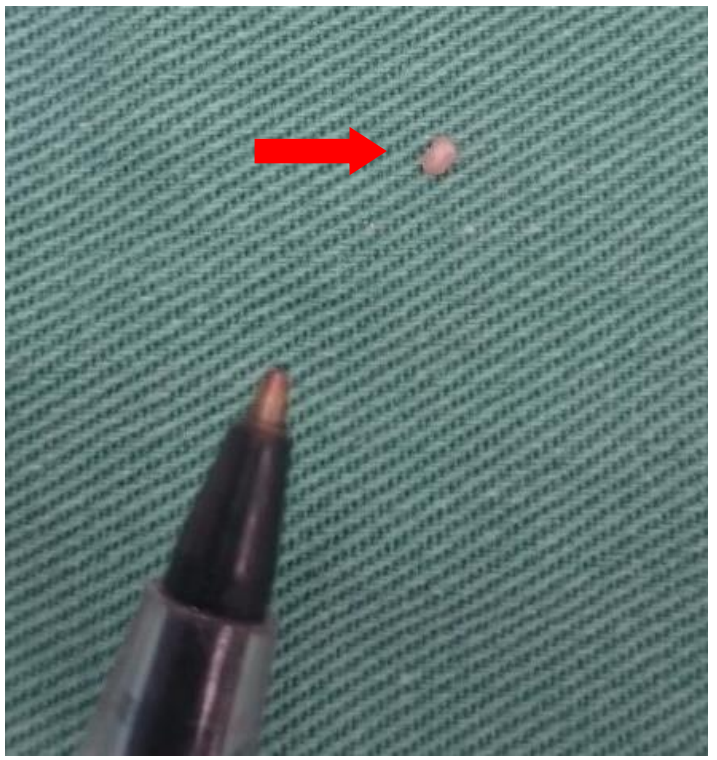

Figura 6. Tampão responsável pela obstrução removido após a amputação peniana (seta).

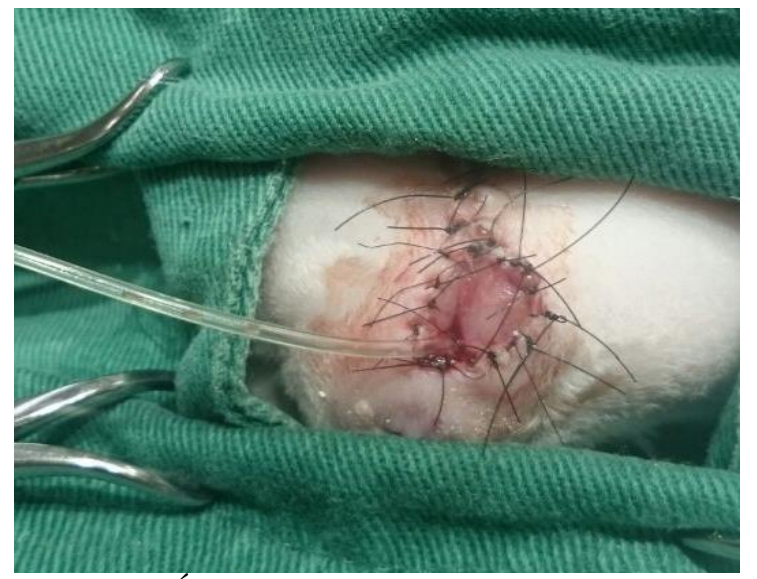

Figura 7. Óstio uretral em região perinealapós uretrostomia.Fonte: Solimar Dutra da Silveira (2016).

\section{Resultados e Discussão}

De acordo com Dibartola \& Westropp (2015), anatomicamente felinos machos apresentam predisposição a quadros obstrutivos de trato urinário inferior, sendo estes, significativamente correlacionados há presença de urólitos e tampões uretrais, associado ainda a diminuição do diâmetro luminal e da elasticidade da uretra peniana.

O histórico e os sinais clínicos de gatos obstruídos estão intimamente relacionados a duração e o grau do quadro obstrutivo do paciente (Peixoto, 1997; Pauluka, 2008). No presente caso relatado, as alterações apresentadas pelo paciente corroboram com dados de outros autores, que descrevem como principais sinais clínicos: apatia, anorexia, disúria ou polaciúria associada a hematúria, e em casos mais graves sinais clínicos relacionados a azotemia, como crises convulsivas desencadeadas por encefalopatia urêmica (Bartges et al., 1996; Markwell \& Smith, 2003; Cowgill \& Langston, 2011; Dibartola \& Westropp, 2015).

Durante exame ultrassonográfico, foi possível constatar a vesícula urinária distendida emoderada concentração sedimentar intraluminal, fato ao qual justificou a presença de plug obstruindo o canal uretral. Ainda pela ecografia, os rins se apresentavam íntegros e levemente aumentados, sendo que em literatura consultada, tem-se como principal achado perante obstruções de ureter ou uretra, a dilatação de pelve renal ou hidronefrose (Espada et al., 2006).

O diagnóstico foi confirmado perante associação do histórico clínico, exame físico e exame ultrassonográfico de região abdominal. Comprovou-se a dificuldade de micção apresentada pelo paciente, como relatado pela tutora, além de distensão vesical e completa obstrução uretral, identificada durante procedimento de sondagem uretral.

O paciente foi diagnosticado e encaminhado para intervenção cirúrgica com três meses de idade devido ao quadro ser caracterizado como emergência (Rabelo et al., 2005). Sendo que, muitos autores não recomendam a realização de qualquer procedimento cirúrgico antes dos dois meses de vida (Hafez, 2004; Björling \& Costa, 2008). A técnica cirúrgica de uretrostomia empregada ao paciente, tinha como principal objetivo o alívio da obstrução, a correção de efeitos sistêmicos como um quadro de uremia e a prevenção de uma recidiva obstrutiva (Dieringer \& Lees, 1991; Ling, 1996).

No presente trabalho, o paciente veio a óbito devido intercorrências anestésicas, onde após duas paradas respiratórias e duas cardiorrespiratórias impossibilitaram o restabelecimento vital, mesmo após a realização de diversas manobras de ressuscitação. Tal desfecho clínico corrobora com percentuais descritos em literatura, onde afirmam que apenas 9,6\% dos felinos sobrevivem após ocorrência de uma parada cardiorrespiratória (Wingfield \& Van Pelt, 1992). 


\section{Conclusão}

A ocorrência de obstrução uretral em felinos é vista como uma enfermidade do aparelho urogenital de grande incidência em medicina veterinária, sendo classificada como urgência ou emergência.

O presente relato contribui de forma relevante na abordagem terapêutica de um paciente jovem com obstrução uretral, uma vez que há poucos relatos publicados relacionados a neonatologia sobre tal enfermidade. A evolução favorável do quadro clínico pode ser muitas vezes atribuída a abordagem inicial do paciente, em razão da associação entre anamnese, exames físico geral e específico detalhado e a escolha correta de métodos complementares de diagnóstico, permitindo assim diferenciar e excluir outras enfermidades e a adoção terapêutica adequada que proporcione a rápida recuperação do paciente, garantindo posteriormente uma melhora na qualidade de vida do mesmo.

\section{Referências}

Alves, A. M. (2006). Tratamento clínico e cirúrgico de obstrução uretral em doença do trato urinário inferior dos felinos. Monografia de Pós-Graduação Lato Sensuem Clínica médica e cirúrgica em pequenos animais da Universidade Castelo Branco. Universidade Castelo Branco, Rio de Janeiro. p.32.

Aquino Filho, R. C. D. (2014). Ressuscitação cérebro-cardiopulmonar em cães e gatos. Monografia de Conclusão do curso de Medicina Veterinária da Faculdade de Agronomia e Medicina Veterinária da Universidade de Brasília. Universidade de Brasília, Brasília DF, p.42.

Bartges, J. W., Finco, D. R., Polzin, D. J., Osborne, C. A., Barsanti, J. A. \& Brown, S. A. (1996). Pathophysiology of urethral obstruction. Veterinary Clinics of North America: Small Animal Practice, 26:255-264.

Bjorling, D. E. \& Costa, G. T. M. (2008). Cirurgia da uretra. In: Slatter, D.H. Manual Saunders de Clínica de Pequenos Animais.2. ed. São Paulo: Roca, p.954-956.

Belone, E. N. S. (2002). Terapêutica do sistema renal em pequenos animais. In: Andrade, F.S. Manual de terapêutica veterinária. 2.ed. São Paulo: Roca, cap. 14, p.285-295.
Corgozinho, B. \& Souza, H. J. M. (2004). Condutas na desobstrução uretral. In: Conferência Sul-Americana de Medicina Veterinária, Anais. $4^{\mathrm{a}}$ ed. Rio de Janeiro.

Cowgill, L. D. \& Langston, C. (2011). Acute kidney insufficiency. In: Bartges, J. W. \& Polzin, D. J. Nephrology and Urology of Small Animals. West Sussex, Ingland: WileyBlackwell, cap. 49, p. 472-514.

Dieringer, T. M. \& Lees, G. E. (1991). The role of perineal urethrostomy in the management of lower urinary tract obstruction. In: August Jr. L. Consultations in feline internal medicine. Philadelphia: 1991, W. B Saunders, Company, p.327-332.

Dibartola, S. P. \& Westropp, J. L. (2015). Doenças do Trato Urinário. In: Nelson, R. \& Couto, C. G. Medicina interna de pequenos animais. $5^{\mathrm{a}}$ ed. São Paulo: Elsevier, p.698-702.

Espada, Y., Novellas, R. \& Gopegui, R. R. (2006). Renal ultrasound in dogs and cats. Veterinary research Communications, 30: 133-137.

Fossum, T. W. (2015). Cirurgia dos sistemas reprodutivo e genital. In: Cirurgia de pequenos animais. $4^{\mathrm{a}}$. ed. São Paulo: Elsevier, p.780855.

Gregory, C. R. (1987). The effects of perineal urethrostomyon urethral function in male cats. The Compendium on Continuing Education for the Practicing Veterinarian: Lawrenceville. 9:895-899.

Hafez, E. S. E. (2004). Distúrbios reprodutivos nos machos. In: Reprodução Animal. $7^{\mathrm{a}}$.ed. São Paulo: Manole, p. 291-301.

Lazzarotto, J. J. (2001). Doença do trato urinário inferior dos felinos associada aos cristais de estruvita - revisão. Revista Faculdade Zootecnia, Veterinária e Agronomia de Uruguaiana, 7/8:55-58.

Ling, G. V. (1996). Enfermedades del aparato urinario de perros y gatos: diagnóstico, tratamiento médico, prevención. Inter-Médica, Buenos Aires, p.233.

Markwell, J. P. \& Smith, H. E. B. (2003). Doença do trato urinário inferior em gatos. Nosso Clínico, v. 6, n. 35, p. 23-26.

Osborne, C. A., Kruger, J. M. \& Lulich, J. P. (1995). Feline lower urinary tract diseases. In: Ettinger, S. J. \& Feldman, E. C. Textbook of Veterinary Internal Medicine. $4^{\mathrm{a}}$. ed. 
Philadelphia: Saunders, v. 2, cap.140, p.18051832.

Osborne, C. A., Caywood, D. D., Johnston, G. R., Polzin, D. J., Lulich, J. P., Kruger, J. M. \& Ulrich, L. K. (1996). Feline perineal urethrostomy: a potential cause of feline lower urinary tract disease. Veterinary Clinics of North America: Small Animal Practice, p.535549.

Pauluka, S. (2008). Obstrução Uretral em Felinos. Monografia de Pós-graduação Lato Sensu em clínica médica e cirúrgica de pequenos animais da Universidade Castelo Branco. Universidade Castelo Branco, Curitiba. 2008, p.21.

Peixoto, E. C. T. M. (1997). Uretrostomia peniana e perineal em felinos domésticos. Revista Ciência Rural, 27(4): 629-633.

Rabelo, R. C., Soares, J. A. \& Leite, R. M. C. (2005). Abordagem otimizada do felino obstruído. In: Fundamentos de Terapia Intensiva Veterinária em Pequenos Animais: condutas no paciente crítico. $1^{\text {a }}$. ed. Rio de Janeiro: LF livros.

Reche Jr, A., Hagiwara, M. K. \& Mamizuka, E. (1998). Estudo clínico da doença do trato inferior em gatos domésticos de São Paulo. Brazilian Journal of Veterinary Research and Animal Science, 35:69-74.

Rossi, C. N., Oliva, V., Matsubara, L. M. \& Serrano, A. C. M. (2007). Ressuscitação cardiorrespiratória em cães e gatos - revisão de literatura. Revista Portuguesa de Ciências Veterinárias, 197-205.

Serrano, S. (2011). El gato obstruido: haciael $100 \%$ de supervivencia. In: Congreso Latinoamericano de Emergencia y Cuidados Intensivos. Anais. Santiago de Chile.

Souza, M. J. H. (1998). Condutas na desobstrução uretral In: Souza, M. J. H. Coletânea em medicina e cirurgia felina. Rio de Janeiro: AS Livros de Veterinária, 67-88.

Wakil, M. F., Martorelli, C. R., Moskoll, P. E. \& Kogikal, M. M. (2010). Classificação em estágios da doença renal crônica em cães e gatos-abordagem clínica, laboratorial e terapêutica. Revista Ciência Rural, 40, p.10.

Wingfield, W. E. \& Van Pelt, D. R. (1992). Respiratory and cardiopulmonary arrest in dogs and cats: 265 cases (1986- 1991). Journal of American Veterinary Medical Association, n $200, \mathrm{p} .1993-1996$.

\section{Article History:}

Received 21 July, 2016

Accepted 13 August, 2016

Available on line 27 September 2016

License information: This is an open-access article distributed under the terms of the Creative Commons Attribution License, which permits unrestricted use, distribution, and reproduction in any medium, provided the original work is properly cited. 